\title{
Diagnostic performance between contrast enhancement, proton MR spectroscopy, and amide proton transfer imaging in patients with brain tumors
}

\section{AUTHOR(S):}

Sakata, Akihiko; Fushimi, Yasutaka; Okada, Tomohisa; Arakawa, Yoshiki; Kunieda, Takeharu; Minamiguchi, Sachiko; Kido, Aki; Sakashita, Naotaka; Miyamoto, Susumu; Togashi, Kaori

\section{CITATION:}

Sakata, Akihiko ... [et al]. Diagnostic performance between contrast enhancement, proton MR spectroscopy, and amide proton transfer imaging in patients with brain tumors. Journal of magnetic resonance imaging : JMRI 2017, 46(3): 732-739

\section{ISSUE DATE:}

2017-09

URL:

http://hdl.handle.net/2433/244235

\section{RIGHT:}

This is the peer reviewed version of the following article: ['JMRI' 46(3) 732-739], which has been published in final form at https://doi.org/10.1002/jmri.25597. This article may be used for non-commercial purposes in accordance with Wiley Terms and Conditions for Use of Self-Archived Versions.; The full-text file will be made open to the public on 14 August 2018 in accordance with publisher's 'Terms and Conditions for Self-Archiving'.; この論文は出版社版でありません。引 用の際には出版社版をご確認ご利用ください。; This is not the published version. Please cite only the published version. 
1 Title

2 Diagnostic Performance between Contrast Enhancement, Proton MR Spectroscopy and

3 Amide Proton Transfer Imaging in Patients with Brain Tumors

4

5 
1 ABSTRACT

2 Purpose

3 To explore the relationship among parameters of magnetic resonance spectroscopy

4 (MRS) and amide proton transfer (APT) imaging, and to assess the diagnostic

5 performance of MRS and APT imaging for grading brain tumors in comparison with

6 contrast enhancement of conventional MRI for preoperative grading in patients with

7 brain tumor.

$8 \quad$ Materials and Methods

9 Institutional review board approval and written informed consent were obtained.

10 Forty-one patients with suspected brain tumors were enrolled in the study. Single-voxel

11 MRS and two-dimensional APT imaging of the same slice level were conducted using a

12 3-T MRI scanner. Positive or negative contrast enhancement on T1-weihted images was

13 assessed by two neuroradiologists. Correlations among metabolite concentrations,

14 metabolite ratios, and calculated histogram parameters, including mean APT (APT mean)

15 and the 90th percentile of APT (APT 90 ) were assessed using Spearman's correlation

16 coefficient. Diagnostic performance was evaluated with receiver operating characteristic

17 (ROC) curve analysis for contrast enhancement, MRS and APT imaging. Values of $\mathrm{p}<$

180.05 were considered statistically significant. 
1 Results

2 Positive correlations with statistical significance were found between total concentration

3 of Cho and $\mathrm{APT}_{90}(\mathrm{r}=0.49)$, and between $\mathrm{Cho} / \mathrm{Cr}$ and $\mathrm{APT}_{\text {mean }}(\mathrm{r}=0.65)$ as well as $\mathrm{APT}_{90}$

4 ( $\mathrm{r}=0.49)$. A negative correlation with statistical significance was observed between

$5 \mathrm{NAA} / \mathrm{Cr}$ and $\mathrm{APT}_{\text {mean }}(\mathrm{r}=-0.52)$. According to ROC curves, Cho/Cr, $\mathrm{APT}_{\text {mean, }} \mathrm{APT}_{90}$,

6 demonstrated higher AUC values than that of contrast enhancement in grading gliomas.

\section{Conclusions}

8 Significant correlations were observed between metabolite concentrations and ratios on

9 MRS and APT values. MRS and APT imaging showed comparable diagnostic capability

10 for grading brain tumors, suggesting that both MRS and APT imaging offer potential for

11 quantitatively assessing similar biological characteristics in brain tumors on non-contrast MRI.

$15 \quad$ Key Words

16 Amide proton transfer imaging;

17 Chemical exchange saturation transfer;

18 Magnetic Resonance Imaging; 
1 Magnetic Resonance Spectroscopy; 
contrast agent has been accepted as a standard method for the characterization of brain contrast enhanced (CE) T1-weighted imaging is used as a measure of brain tumor

6 malignancy and grading. ${ }^{1}$ However, the accuracy of this approach is reportedly

7 limited. ${ }^{2,3}$ Moreover, with the recent availability of anti-angiogenic pharmacotherapies

8 such as bevacizumab and other agents, ${ }^{4,5}$ evaluating the status of brain tumors has been

9 complicated by the protection of the BBB afforded by these treatment. ${ }^{6}$

11 tumors without using contrast media. Among these, ${ }^{1} \mathrm{H}$-magnetic resonance spectroscopy (MRS) has been established as a technique providing information on

13 chemical metabolites present in living tissues. ${ }^{7}$ Amide proton transfer (APT) imaging, a novel chemical exchange saturation transfer (CEST)-based molecular MRI technique,

15 has recently been introduced to the clinical setting. ${ }^{8-12}$ APT imaging is designed to

16 detect endogenous mobile proteins and peptides in tissues. ${ }^{13}$ Both MRS and APT

17 imaging provide metabolic information that can be exploited to study the biological 18 behaviors of tumors. ${ }^{14,15}$ 
1

2 similarities and correlations and would provide important oncologic information. To

3 date, several studies have demonstrated that these advanced techniques have the

4 potential to characterize brain tumors. ${ }^{7-12,16-18}$ However, very few clinical studies have

$5 \quad$ discussed correlations between findings from MRS and APT imaging. ${ }^{19}$

6

7 obtained from MRS and APT imaging and 2) assess the diagnostic performance of APT

We assumed that APT imaging and metabolites identified on MRS would show The objectives of this study were to: 1) explore relationships among parameters imaging and MRS for grading brain tumors in comparison with contrast enhancement of conventional MRI.

\section{MATERIALS AND METHODS}

\section{Patients}

The institutional review board of our university approved this study. Written informed consent was obtained from all patients or their guardian/s. Between May 2013 and August 2014, a total of 41 consecutive patients with suspected brain tumor and symptoms corresponding to World Health Organization (WHO) performance status $\leq 2$ were enrolled in the study. The following inclusion criteria were used: a) MRS and APT imaging performed during the same examination; and b) presence of treatment-naïve or 
1 recurrent tumor with histological verification. Exclusion criteria were: a) degradation of

2 image quality due to susceptibility artifacts or motion artifacts; b) unsatisfactory

3 shimming such as a larger full width at half maximum (FWHM) of water peak reaching

$4>0.10 \mathrm{ppm}$; or c) extra-axial tumors such as meningioma. When motion artifacts were

5 suspected, original series of APT imaging were visually inspected if there are apparent

6 movements of head position during image acquisition. Five patients were excluded due

7 to severe motion artifacts during APT imaging (a), 5 patients were excluded due to the

8 FWHM criterion (b), and 4 patients with lymphoma, one patient with brain metastasis

9 from melanoma, 5 patients with meningioma were also excluded (c). A final total of 21

10 patients were thus included in this study.

MR Imaging

MR Spectroscopy

All MRI was performed using a whole-body 3-T system (Vantage; Toshiba

Medical Systems, Otawara, Japan) with a 32-channel head coil. MRS was obtained

using a single-voxel point-resolved spectroscopic sequence with water suppression pulse and the following parameters: repetition time (TR), 2000 ms; echo time (TE), 136 ms; flip angle, $90^{\circ}$; and scan time, 4 min $18 \mathrm{~s}$. The volume of interest (VOI) was placed 
1 on the area showing the solid portion of tumor on T2-weighted imaging, and the cystic

2 or necrotic portion and hemorrhagic regions were carefully avoided. Size of the VOI

3 varied from $15 \times 15 \times 15 \mathrm{~mm}^{3}$ to $20 \times 20 \times 20 \mathrm{~mm}^{3}$, depending on tumor size.

MR data were transferred to a workstation, then MRS was analyzed using

5 LCModel version 6.3-0C software (LCModel, Ontario, Canada) using the basis set.

6 Analysis was undertaken on water-suppressed spectral domain data for chemical shifts

7 in the range of $0.0-4.0 \mathrm{ppm}$. Absolute concentrations of N-acetyl aspartate (NAA),

8 creatine ( $\mathrm{Cr}$ ) and choline (Cho), and NAA to creatine ratio (NAA/Cr) and choline to

9 creatine $(\mathrm{Cho} / \mathrm{Cr})$ ratios were calculated using known concentrations of tissue water. ${ }^{20}$

APT imaging

APT imaging was conducted with a 2-dimensional fast advanced spin echo sequence, which is similar to the half-Fourier acquisition single-shot turbo spin-echo sequence. The imaging slice was set at the midpoint of the VOI of MRS, which enabled values of APT to be obtained at the VOI of MRS. The following parameters were used in a single-slice axial acquisition: field of view, $230 \times 230 \mathrm{~mm}$; matrix, $256 \times 256$; TR, 9000 ms; TE, $60 \mathrm{~ms}$; flip angle, $90^{\circ}$; refocusing flip angle, $160^{\circ}$; slice thickness, $5 \mathrm{~mm}$; 
1 bandwidth, $651 \mathrm{~Hz} /$ pixel; saturation power, flip angle 500 ${ }^{\circ}(1 \mu \mathrm{T})$;

2 magnetization-transfer (MT) Contrast pulse shot, 25; inter-pulse delay, $1 \mathrm{~ms}$; and scan

3 time, 6 min $9 \mathrm{~s}$. MT spectra over an offset range of $\pm 10 \mathrm{ppm}$ with a step size of $0.5 \mathrm{ppm}$

4 with respect to water resonance were obtained.

$6 \quad$ Imaging Analysis

7 Conventional MRI

Two board-certified neuroradiologists (_._. and _... with 8 and 17 years of

9 experience) independently analyzed imaging characteristics of brain tumor. They assessed if contrast enhancement is present $(\mathrm{CE}+\mathrm{ve})$ or not (CE -ve) on CE

11 T1-weighted image of patients with brain tumor.

APT imaging

The minimum value for MT spectra obtained from APT imaging was estimated from the original data by spline interpolation with Lorentzian function fitting, and displacement from the water resonance frequency owing to B0-field inhomogeneity was corrected. For quantification, asymmetric magnetization transfer ratio (MTR asym) analysis was performed with respect to the water resonance frequency on the 
1 shift-corrected z-spectrum fit between a parameter for amide proton exchange and its

2 symmetrically opposite side, such that $\operatorname{MTR}_{\text {asym }}(+\Delta \omega)=\operatorname{MTR}(-\Delta \omega)-\operatorname{MTR}(+\Delta \omega)$,

3 where MTR is the magnetization transfer ratio and $\Delta \omega$ is the frequency (in parts per

4 million) of amide and the water exchange site with respect to water (typically between

$5 \quad 3.3$ and $3.7 \mathrm{ppm}$ ) obtained from the shift-corrected z-spectrum. The VOI of MRS was

6 automatically transferred to the APT image of each patient using an in-house script in

7 Matlab 2013b (The MathWorks, Natick, MA) (Fig. 1). Finally, mean MTR asym $_{\text {ng }}$

8 (APT $\left._{\text {mean }}\right)$, the 90th percentile of $\mathrm{MTR}_{\mathrm{asym}}\left(\mathrm{APT}_{90}\right)$, standard deviation, kurtosis and

9 skewness of VOIs were calculated.

Histologic Evaluation

Pathological diagnosis was determined according to WHO 2007 criteria by a board-certified neuropathologist. ${ }^{21}$ Grade 1 and 2 were regarded as low grade, while grade 3 and 4 were regarded as high grade.

Statistical Analysis

Data were checked by Gaussian distribution and homogeneity testing before analysis. Intra-rater agreements were assessed with Cohen's kappa. If there were 
1 disagreement between the two raters, third radiologist (_._., a board-certified

2 neuroradiologist with 24 years of experience) made final decision. Correlations between

3 parameters obtained from MRS and APT imaging were analyzed using Spearman

4 correlation analysis. Receiver-operating characteristic (ROC) analyses were conducted

5 to determine optimal thresholds, as well as sensitivity and specificity for grading brain

6 tumors with each technique. We used conventional MRI characteristics as reference

7 standards. Areas under the curve (AUCs) were statistically compared using a method

8 described by Delong et al. ${ }^{22}$

All statistical analyses were conducted using Stata version 13 software (Stata

Corp, Texas, US). Values of $\mathrm{p}<0.05$ were considered statistically significant.

RESULTS

\section{Patients}

Patient population was summarized in Table 1. Patients comprised 13 males and 8 females (mean age, 50.0 \pm 20.2 years; range, 11-85 years). Tumors comprised 21

16 gliomas (grade II, $n=10$; grade III, $n=3$; grade IV, $n=8$ ). Two patients were diagnosed

17 with recurrent gliomas. Typical cases are shown in Fig. 1 and Fig. 2. 


\section{Intra-rater agreement of conventional MRI analyses by neuroradiologists}

CE +ve was diagnosed in 16 patients, and CE -ve was diagnosed in 5 patients

(Table 1). Cohen’s kappa was 1.0 and agreement was 100\%.

\section{Correlations between MRS and APT imaging}

Correlations between among metabolites concentration or ratio from MRS and

$\mathrm{APT}_{\text {mean }}$ or $\mathrm{APT}_{90}$ are summarized in Table 2. Positive correlations with statistical significance were found between Cho and $\mathrm{APT}_{90}$, and between $\mathrm{Cho} / \mathrm{Cr}$ and $\mathrm{APT}_{\text {mean }}$. Negative correlations with statistical significance were observed between NAA/Cr and $\mathrm{APT}_{\text {mean. }}$ Negative correlations were observed between NAA and $\mathrm{APT}_{\text {mean }}(\mathrm{r}=-0.43$, $\mathrm{p}=0.05$ ). Standard deviation, skewness and kurtosis of $\mathrm{APT}_{\text {asym }}$ showed no significant correlations with Cho, NAA Cr, or its ratios (Table. 2).

Diagnostic capability for grading brain tumors on contrast enhancement, MRS and

\section{APT imaging}

ROC curves illustrating the performance of contrast enhancement, Cho, Cho/Cr, $\mathrm{APT}_{\text {mean }}, \mathrm{APT}_{90}$ are shown in Fig. 3. According to ROC curves, the $\mathrm{APT}_{\text {mean }}$ cutoff of 2.72 offered the best combination of sensitivity (72.7\%) and specificity (90\%) in the 
1 characterization of high-grade gliomas, while the $\mathrm{APT}_{90}$ cut-off of 6.61 demonstrated

2 the optimal combination of sensitivity (90.9\%) and specificity (70\%). sensitivity (63.6\%) and specificity (100\%) among total concentration of metabolites.

5 Among the ratios of the two metabolites above, the Cho/Cr threshold value of 0.50

6 generated the best combination of sensitivity (100\%) and specificity (93.8\%) in the

7 discrimination of high- and low-grade tumors. AUCs of each parameter were

8 summarized in Table. 3. All the imaging parameters above except Cho demonstrated

9 higher AUC values than that of contrast enhancement (AUC was 0.65). None of the

10 AUCs obtained from these variables showed significant differences (Fig. 3, APT $_{90}$ vs

$11 \mathrm{APT}_{\text {mean, }} \mathrm{P}=0.74, \mathrm{APT}_{90}$ vs $\mathrm{Cho} / \mathrm{Cr}, \mathrm{P}=0.35$, Cho/Cr vs $\mathrm{APT}_{\text {mean }} \mathrm{P}=0.72$ ).

\section{DISCUSSION}

We made two important observations in this study. First, we found significant moderate correlations between the concentration of metabolites and $\mathrm{APT}_{\text {asym }}$ parameters.

16 Few studies have investigated the associations among MRS and CEST. In the literature,

17 lactate and $\mathrm{MTR}_{\mathrm{asym}}$ at $3.5 \mathrm{ppm}$ correlated well with infarcted area in induced middle

18 cerebral artery occlusion, ${ }^{23}$ and a good correlation was seen between CEST at 2 ppm 
1 and creatine concentration for implanted gliosarcoma cells. ${ }^{24}$ However, those studies

2 used rat models, and only one clinical study has investigated correlations between MRS

3 and CEST in human brain tumors. ${ }^{19}$ Some investigators prefer $\mathrm{APT}_{90}$ or other variables

4 close to maximum values over mean APT values, ${ }^{8,19}$ because the $90 \%$ histogram cutoff

5 is less influenced by random statistical fluctuations, and is thus considered superior to

6 the maximum value. ${ }^{25}$ When comparing Cho and $\mathrm{APT}_{90}$, a positive correlation was seen

7 in patients with intra-axial brain tumors, supporting the recent results from Park et al. ${ }^{19}$

8 These findings suggest the feasibility of quantitatively assessing similar biological

9 characteristics in brain tumors using MRS and APT imaging as different modalities.

10 Such correlation is unlikely to be due to signals from Cho, because the CEST effect at

113.5 ppm from endogenous metabolites including Cho and NAA might not be visible at

$123 \mathrm{~T} .{ }^{26}$ Generally, malignant tumor cells show high proliferative activity and cellularity.

13 The Cho peak corresponds to increased cell attenuation and membrane turnover in

14 neoplastic tissues. ${ }^{27,28}$ APT values have recently been reported to show moderate

15 correlations with cell density or MIB-1 index, as a representative marker for

16 proliferative activity of the brain tumor. ${ }^{9}$ A possible explanation for the positive

17 correlation between Cho and $\mathrm{APT}_{90}$, therefore, involves the proliferative activity and

18 cellularity of tumor cells. 
2 predominantly located in neurons and is thus decreased in all neoplasms that cause

3 neurons to be displaced or replaced with malignant cells. ${ }^{29}$ Numerous studies have

4 demonstrated decreased NAA peak, mainly in glial neoplasms ${ }^{30}$ and primary CNS

5 lymphoma, ${ }^{31}$ consistent with our results. Our results also suggest that $\mathrm{APT}_{\text {mean }}$ indirectly

6 reflects neuronal loss due to brain tumor infiltration. To testify our hypothesis, further

$7 \quad$ study including stereotactic biopsy based on the APT image should be needed. nor between NAA and $\mathrm{APT}_{90}$. A possible explanation for this discrepancy is that $\mathrm{APT}_{90}$ and $\mathrm{APT}_{\text {mean }}$ may be different biomarkers, and similar discrepancies were reported between minimum and mean apparent diffusion coefficient from diffusion-weighted imaging. ${ }^{32}$ Jiang et al. recently showed that the maximum value from APT imaging was significantly lower in patients with primary CNS lymphoma than in patients with

14 Glioblastoma, while $\mathrm{APT}_{\text {mean }}$ values did not show any such significant differences between patient groups. ${ }^{12}$ To clarify and verify different information for these parameters, further studies are needed. Second, MRS and APT imaging showed comparable diagnostic performance in differentiating between high- and low-grade brain tumors. Several studies have shown a 
1 significant increase in $\mathrm{Cho} / \mathrm{Cr}$ in high-grade tumors compared with that of low-grade

2 tumors at long TE with clinical scanners. ${ }^{17,18}$ Past studies also demonstrated that the

3 utility of amount of metabolite or metabolite concentration ratio in grading brain

4 tumor. $^{33}$ Recently, $\mathrm{APT}_{\text {asym }}$ values are reportedly suitable for differentiating between

5 high- and low-grade brain tumors, mainly in adult populations. ${ }^{8-11}$ The diagnostic

6 capability of MRS and APT imaging for grading brain tumors was investigated and

7 compared with that of conventional MRI in this study. We found that MRS and APT

8 imaging shows higher AUCs compared with that of grading diagnosed by

9 neuroradiologists based on conventional MRI. Although conventional MRI including

10 CE-T1 weighted image has been widely used in pre- and post-operative assessment of

11 brain tumor, several researchers reported its limited capability of glioma-grading., ${ }^{2,3}$ Our

result may suggest that these two imaging sequences can give us additional information of brain tumor even without contrast materials.

Interestingly, metabolite ratios to creatine showed either better correlation with APT value or diagnostic capability of glioma grading than the concentration. The reason

16 is hard to explain. Previous study suggested that measuring concentration of metabolites

17 in brain tumor might be difficult, especially in high-grade glioma. ${ }^{34}$ Metabolite ratios

18 are frequently used in MRS studies and assumed as an internal standard, can give us 
1 more accurate information in grading glioma than absolute concentration. ${ }^{35}$ Our results

2 may be in line with this. current study, the origin of APT signal still remains unclear. Many researchers have

5 demonstrated some biological signature such as cytosolic protein including folding

6 status or amount of specific protein contributes APT signal. ${ }^{36,37}$ Furthermore, there are

7 some discussions about the confounding effects of asymmetry of the solid-phase MT

8 effect and possible intra- and intermolecular nuclear Overhauser effects (NOE) of

9 aliphatic protons and T1 value of the tissue to APT-weighted image. ${ }^{38}$ Some authors

10 implied or demonstrated that the background MT asymmetry or NOE effects are

11 negligible on $3 \mathrm{~T}^{10}$ while others argue that MT asymmetry is more significant than APT

12 effect even on 3 T. $^{38}$ According to the recent researches with ultra-high field MRI, NOE

13 imaging has potential to delineate portion with high cellularity, or BBB disruption in

14 glioma without contrast agents. ${ }^{39,40}$ MT contrast in the brain tumor has been reported to

15 be decreased compared with white matter, and correlate with nuclear density. ${ }^{41}$ To

16 investigate the association among NOE, MT asymmetry and metabolites in brain tumors,

17 further studies at ultra-high field MRI may be needed. Environmental factors such as

$18 \mathrm{pH}$ and temperature also have great effects on CEST image. $\mathrm{pH}$ imaging, which is a 
1 cutting-edge application of CEST, and may become a potential marker of hypoxia. ${ }^{42}$

2 Recent study showed the promising result of amine-proton based CEST images, which

3 is focusing on $3 \mathrm{ppm}$, in detecting intratumoral $\mathrm{pH}$ changes. ${ }^{43}$ However, this effect is

4 somewhat confusing in the assessment of brain tumor with APT images; High-grade

5 tumors with high proliferation activity can show acidotic changes due to anaerobic

6 metabolism or necrosis, which causes lactate peak in MRS. ${ }^{44}$ However, such acidotic

7 change decreases the rate of proton exchange, and theoretically lessen the signal of

8 tumors on APT image. Longo et al recently demonstrated this "Warburg effect" in the

9 breast cancer model mice, using two-different modalities such as positron emission

10 tomography and pH-oriented CEST image with iopamidol as an exogenous CEST

11 contrast agent. ${ }^{45}$ APT image combined with MRS, especially chemical shift imaging

12 including lactate peak may also offer complementary spatial information of tumor

13 microenvironment including $\mathrm{pH}$ and metabolism. Recent post-processing advance, such

14 as MT and relaxation-normalized APT, may give us additional information about

15 intratumoral $\mathrm{pH}$ change. ${ }^{46}$ Second, we did not adjust for APT imaging using controls,

16 such as contralateral normal-appearing white matter. Such adjustment might be useful

17 for minimizing the effects of native MTR $_{\text {asym }}$ discussed above. However, several studies

18 have shown that diagnostic capability did not differ between imaging with and without 
1 such adjustment. ${ }^{9,11}$ In addition, some high-grade tumors may invade diffusely, leaving

2 little normal-appearing white matter. ${ }^{47}$ Third, some cases with glioblastoma showed

3 relatively high Cramer Rao Lower Bounds (CRLB) (\%SD is more than 15) in NAA

4 value. High grade gliomas often show low NAA peak due to glial cell invasion, as a

5 result, fitting of low amount of total NAA concentration may be difficult. Although

6 NAA with larger CRLB in cases with glioblastoma might be unreliable and less

7 reproducible, exclusion of cases with glioblastoma were not beneficial in this study.

8 Strength of this study was the fact that, to clarify the correlation, volume of interest was

9 placed at the same location as the tumor on both MRS and APT imaging. To verify these

10 correlations, a further study with direct comparison of histology obtained from

11 stereotactic biopsy based on combined analysis using MRS and APT imaging may be

12 warranted.

In conclusion, significant correlations were observed between metabolite

concentrations and ratios on MRS and APT values. MRS and APT imaging showed comparable diagnostic capability for grading brain tumors, suggesting that both MRS and APT imaging offer potential for assessing the proliferative activity of brain tumors on non-contrast MRI. 
Table 1. Patient Characteristics

\begin{tabular}{|c|c|c|c|c|c|c|c|c|c|c|c|c|c|}
\hline Case & Age & Sex & $\begin{array}{l}\text { Histology } \\
\text { Diagnosis }\end{array}$ & $\begin{array}{c}\text { Histology } \\
\text { Grade }\end{array}$ & CE & Cho & NAA & $\mathrm{Cr}$ & $\begin{array}{c}\mathrm{APT}_{\text {mean }} \\
\text { (\%) }\end{array}$ & $\begin{array}{l}\mathrm{APT}_{90} \\
(\%)\end{array}$ & $\begin{array}{l}\text { APT } \\
\text { std }\end{array}$ & $\begin{array}{c}\text { APT } \\
\text { skewness }\end{array}$ & $\begin{array}{c}\text { APT } \\
\text { kurtosis }\end{array}$ \\
\hline 1 & 57 & $\mathrm{M}$ & DA & 2 & +ve & 1.86 & 4.65 & 5.92 & 2.03 & 8.18 & 0.11 & -0.24 & 3.28 \\
\hline 2 & 70 & F & AA & 3 & +ve & 3.60 & 3.91 & 5.23 & 4.31 & 7.16 & 0.05 & 0.56 & 3.18 \\
\hline 3 & 35 & $\mathrm{M}$ & Oligo & 2 & -ve & 1.89 & 3.64 & 4.69 & 0.05 & 2.74 & 0.05 & 0.19 & 3.03 \\
\hline 4 & 49 & $\mathrm{M}$ & $\mathrm{AO}$ & 3 & -ve & 1.84 & 2.60 & 3.68 & 0.99 & 5.61 & 0.11 & -0.67 & 3.75 \\
\hline 5 & 72 & $\mathrm{M}$ & GBM & 4 & +ve & 8.66 & 3.55 & 4.39 & 3.28 & 7.26 & 0.09 & -0.22 & 3.73 \\
\hline 6 & 71 & $\mathrm{M}$ & DA & 2 & -ve & 0.92 & 2.99 & 4.79 & -1.36 & 0.41 & 0.04 & 0.09 & 2.46 \\
\hline 7 & 44 & F & Oligo & 2 & +ve & 1.31 & 2.21 & 3.54 & 2.46 & 6.20 & 0.06 & 0.13 & 2.49 \\
\hline 8 & 27 & M & GBM & 4 & +ve & 0.79 & 0.56 & 0.70 & 3.55 & 6.61 & 0.07 & -0.14 & 2.41 \\
\hline
\end{tabular}




\begin{tabular}{|c|c|c|c|c|c|c|c|c|c|c|c|c|c|}
\hline 9 & 27 & $\mathrm{M}$ & OA & 2 & +ve & 1.28 & 0.72 & 2.63 & 3.47 & 7.61 & 0.05 & 0.38 & 2.80 \\
\hline 10 & 62 & F & GBM & 4 & +ve & 0.87 & 0.00 & 0.67 & 3.51 & 6.69 & 0.04 & 0.29 & 3.28 \\
\hline 11 & 67 & $\mathrm{M}$ & DA & 2 & -ve & 1.81 & 4.13 & 5.20 & 0.23 & 1.72 & 0.04 & 0.08 & 2.51 \\
\hline 12 & 64 & F & GBM & 4 & +ve & 12.83 & 0.20 & 6.23 & 2.72 & 7.75 & 0.07 & 0.42 & 5.09 \\
\hline 13 & 80 & M & DA & 2 & +ve & 1.12 & 3.36 & 4.98 & 0.60 & 5.43 & 0.13 & -0.81 & 3.00 \\
\hline 14 & 11 & F & AA & 3 & +ve & 15.10 & 4.02 & 10.62 & 5.46 & 7.95 & 0.06 & -0.22 & 2.66 \\
\hline 15 & 24 & $\mathrm{M}$ & Oligo & 2 & +ve & 2.45 & 8.95 & 8.99 & -0.31 & 5.13 & 0.08 & 0.04 & 3.84 \\
\hline 16 & 38 & $\mathrm{M}$ & $\begin{array}{l}\text { GBM } \\
\text { (Rec) }\end{array}$ & 4 & +ve & 6.00 & 0.17 & 6.25 & 4.91 & 10.58 & 0.14 & 0.52 & 2.23 \\
\hline 17 & 44 & M & $\begin{array}{l}\text { Oligo } \\
\text { (Rec) }\end{array}$ & 2 & +ve & 2.48 & 0.00 & 1.25 & 1.99 & 8.76 & 0.20 & -1.22 & 4.61 \\
\hline
\end{tabular}




\begin{tabular}{|c|c|c|c|c|c|c|c|c|c|c|c|c|c|}
\hline 18 & 44 & $\mathrm{~F}$ & $\mathrm{GBM}$ & 4 & $+\mathrm{ve}$ & 3.29 & 0.00 & 3.40 & 2.94 & 6.90 & 0.13 & -0.96 & 3.26 \\
\hline 19 & 34 & $\mathrm{M}$ & $\mathrm{GBM}$ & 4 & $+\mathrm{ve}$ & 1.79 & 1.67 & 2.80 & 0.87 & 7.86 & 0.10 & 0.28 & 3.16 \\
\hline 20 & 85 & $\mathrm{~F}$ & $\mathrm{GBM}$ & 4 & $+\mathrm{ve}$ & 2.85 & 3.66 & 4.48 & -1.75 & 10.65 & 0.24 & -1.27 & 5.10 \\
\hline 21 & 46 & $\mathrm{~F}$ & Oligo & 2 & $-\mathrm{ve}$ & 2.28 & 8.02 & 6.89 & -2.52 & 3.85 & 0.08 & -0.43 & 4.35 \\
\hline
\end{tabular}

std, standard deviation; DA, Diffuse Astrocytoma;AA, Anaplastic Astrocytoma; OA, Oligoastrocytoma; AO, Anaplastic oligoastrocytoma; GBM, Glioblastoma; Oligo, Oligodendroglioma; Rec, recurrence; CE +ve represents positive contrast enhancement, and CE -ve represents negative contrast enhancement; LG, Low-Grade diagnosed by neuroradiologist; HG, High-Grade diagnosed by neuroradiologist. 
Table 2. The correlation among parameters obtained by MRS and APT histogram analysis

\begin{tabular}{|c|c|c|c|c|}
\hline & Cho & NAA & Cho/Cr & NAA/Cr \\
\hline APT $_{\text {mean }}$ & $0.23(\mathrm{p}=0.32)$ & -0.43 & $0.65 *$ & $-0.52^{*}$ \\
& $(\mathrm{p}=0.32)$ & $(\mathrm{p}=0.05)$ & $(\mathrm{p}=0.0015)$ & $(\mathrm{p}=0.016)$ \\
\hline $\mathrm{APT}_{90}$ & $0.49 *$ & -0.31 & $0.59 *$ & -0.37 \\
& $(\mathrm{p}=0.025)$ & $(\mathrm{p}=0.17)$ & $(\mathrm{p}=0.005)$ & $(\mathrm{p}=0.098)$ \\
\hline
\end{tabular}

\begin{tabular}{|c|c|c|c|c|}
\hline & Cho & NAA & Cho/Cr & NAA/Cr \\
\hline $\mathrm{APT}_{\text {std }}$ & 0.33 & -0.19 & 0.13 & 0.06 \\
& $(\mathrm{p}=0.15)$ & $(\mathrm{p}=0.42)$ & $(\mathrm{p}=0.58)$ & $(\mathrm{p}=0.78)$ \\
\hline $\mathrm{APT}_{\text {skewness }}$ & -0.05 & -0.14 & 0.1 & -0.24 \\
& $(\mathrm{p}=0.84)$ & $(\mathrm{p}=0.54)$ & $(\mathrm{p}=0.67)$ & $(\mathrm{p}=0.29)$ \\
\hline $\mathrm{APT}_{\text {kurtosis }}$ & 0.39 & 0.04 & 0.23 & 0.17 \\
& $(\mathrm{p}=0.08)$ & $(\mathrm{p}=0.63)$ & $(\mathrm{p}=0.32)$ & $(\mathrm{p}=0.47)$ \\
\hline
\end{tabular}

std, standard deviation

$* p$ value $<0.05$ 
Table 3. AUC values in ROC analysis for grading glioma with contrast enhancement on T1-weighted imaging, MRS, and APT imaging.

\begin{tabular}{ccc}
\hline Parameters & Area Under the Curve & 95\% Confidence Interval \\
\hline Contrast Enhancement & 0.65 & 0.47 to 0.84 \\
\hline $\mathrm{APT}_{\text {mean }}$ & 0.82 & 0.62 to 1.00 \\
\hline $\mathrm{APT}_{90}$ & 0.77 & 0.54 to 1.00 \\
\hline $\mathrm{Cho}$ & 0.72 & 0.47 to 0.97 \\
\hline Cho/Cr & 0.90 & 0.73 to 1.00 \\
\hline
\end{tabular}




\section{References}

1. Runge VM, Muroff LR, Jinkins JR. Central nervous system: review of clinical use of contrast media. Topics in magnetic resonance imaging : TMRI 2001;12:231-263.

2. Scott JN, Brasher PM, Sevick RJ, Rewcastle NB, Forsyth PA. How often are nonenhancing supratentorial gliomas malignant? A population study. Neurology 2002;59:947-949.

3. Lee EJ, Lee SK, Agid R, Bae JM, Keller A, Terbrugge K. Preoperative grading of presumptive low-grade astrocytomas on MR imaging: diagnostic value of minimum apparent diffusion coefficient. AJNR American journal of neuroradiology 2008;29:1872-1877.

4. de Groot JF, Lamborn KR, Chang SM, et al. Phase II study of aflibercept in recurrent malignant glioma: a North American Brain Tumor Consortium study. J Clin Oncol 2011;29:2689-2695.

5. Schiff D, Kesari S, de Groot J, et al. Phase 2 study of CT-322, a targeted biologic inhibitor of VEGFR-2 based on a domain of human fibronectin, in recurrent glioblastoma. Invest New Drugs $2015 ; 33: 247-253$. 
6. Hygino da Cruz LC, Jr., Rodriguez I, Domingues RC, Gasparetto EL, Sorensen AG. Pseudoprogression and pseudoresponse: imaging challenges in the assessment of posttreatment glioma. AJNR American journal of neuroradiology 2011;32:1978-1985.

7. $\mathrm{Oz}$ G, Alger JR, Barker PB, et al. Clinical proton MR spectroscopy in central nervous system disorders. Radiology 2014;270:658-679.

8. Park JE, Kim HS, Park KJ, Choi CG, Kim SJ. Histogram Analysis of Amide Proton Transfer Imaging to Identify Contrast-enhancing Low-Grade Brain Tumor That Mimics High-Grade Tumor: Increased Accuracy of MR Perfusion. Radiology 2015;277:151-161.

9. Togao O, Yoshiura T, Keupp J, et al. Amide proton transfer imaging of adult diffuse gliomas: correlation with histopathological grades. Neuro-oncology 2014;16:441-448.

10. Zhou J, Zhu H, Lim M, et al. Three-dimensional amide proton transfer MR imaging of gliomas: Initial experience and comparison with gadolinium enhancement. Journal of magnetic resonance imaging : JMRI 2013;38:1119-1128.

11. Sakata A, Okada T, Yamamoto A, et al. Grading glial tumors with 
amide proton transfer MR imaging: different analytical approaches. J Neurooncol 2015;122:339-348.

12. Jiang S, Yu H, Wang X, et al. Molecular MRI differentiation between primary central nervous system lymphomas and high-grade gliomas using endogenous protein-based amide proton transfer MR imaging at 3 Tesla. Euro Radiol 2016;26:64-71.

13. Zhou J, Payen JF, Wilson DA, Traystman RJ, van Zijl PC. Using the amide proton signals of intracellular proteins and peptides to detect pH effects in MRI. Nat Med 2003;9:1085-1090.

14. Zhang Z, Zeng Q, Liu Y, Li C, Feng D, Wang J. Assessment of the intrinsic radiosensitivity of glioma cells and monitoring of metabolite ratio changes after irradiation by 14.7-T high-resolution (1)H MRS. NMR in biomedicine 2014;27:547-552.

15. Sagiyama K, Mashimo T, Togao O, et al. In vivo chemical exchange saturation transfer imaging allows early detection of a therapeutic response in glioblastoma. Proc Natl Acad Sci U S A $2014 ; 111: 4542-4547$.

16. Julia-Sape M, Coronel I, Majos C, et al. Prospective diagnostic 
performance evaluation of single-voxel 1H MRS for typing and grading of brain tumours. NMR in biomedicine 2012;25:661-673.

17. Kim JH, Chang KH, Na DG, et al. 3T $1 \mathrm{H}-\mathrm{MR}$ spectroscopy in grading of cerebral gliomas: comparison of short and intermediate echo time sequences. AJNR American journal of neuroradiology 2006;27:1412-1418.

18. Zeng Q, Liu H, Zhang K, Li C, Zhou G. Noninvasive evaluation of cerebral glioma grade by using multivoxel 3D proton MR spectroscopy. Magnetic resonance imaging 2011;29:25-31.

19. Park JE, Kim HS, Park KJ, Kim SJ, Kim JH, Smith SA. Pre- and Posttreatment Glioma: Comparison of Amide Proton Transfer Imaging with MR Spectroscopy for Biomarkers of Tumor Proliferation. Radiology 2015:142979.

20. Provencher SW. Estimation of metabolite concentrations from localized in vivo proton NMR spectra. Magnetic resonance in medicine 1993;30:672-679.

21. Louis DN, Ohgaki H, Wiestler OD, et al. The 2007 WHO classification of tumours of the central nervous system. Acta Neuropathol 
2007;114:97-109.

22. DeLong ER, DeLong DM, Clarke-Pearson DL. Comparing the areas under two or more correlated receiver operating characteristic curves: a nonparametric approach. Biometrics 1988;44:837-845.

23. Sun PZ, Cheung JS, Wang E, Lo EH. Association between $\mathrm{pH}-$ weighted endogenous amide proton chemical exchange saturation transfer MRI and tissue lactic acidosis during acute ischemic stroke. J Cereb Blood Flow Metab 2011;31:1743-1750.

24. Cai K, Singh A, Poptani H, et al. CEST signal at 2ppm (CEST@2ppm) from Z-spectral fitting correlates with creatine distribution in brain tumor. NMR in biomedicine 2015;28:1-8.

25. Kim HS, Suh CH, Kim N, Choi CG, Kim SJ. Histogram analysis of intravoxel incoherent motion for differentiating recurrent tumor from treatment effect in patients with glioblastoma: initial clinical experience. AJNR American journal of neuroradiology $2014 ; 35: 490-497$.

26. Lee JS, Xia D, Jerschow A, Regatte RR. In vitro study of endogenous CEST agents at $3 \mathrm{~T}$ and $7 \mathrm{~T}$. Contrast media \& molecular imaging 
2015.

27. Gupta RK, Cloughesy TF, Sinha U, et al. Relationships between choline magnetic resonance spectroscopy, apparent diffusion coefficient and quantitative histopathology in human glioma. J Neurooncol 2000;50:215-226.

28. Sabatier J, Gilard V, Malet-Martino M, et al. Characterization of choline compounds with in vitro $1 \mathrm{H}$ magnetic resonance spectroscopy for the discrimination of primary brain tumors. Invest Radiol 1999;34:230-235.

29. Moffett JR, Ross B, Arun P, Madhavarao CN, Namboodiri AM. $\mathrm{N}$-Acetylaspartate in the CNS: from neurodiagnostics to neurobiology. Prog Neurobiol 2007;81:89-131.

30. Usenius JP, Kauppinen RA, Vainio PA, et al. Quantitative metabolite patterns of human brain tumors: detection by $1 \mathrm{H}$ NMR spectroscopy in vivo and in vitro. J Comput Assist Tomogr 1994;18:705-713.

31. Mansour A, Qandeel M, Abdel-Razeq H, Abu Ali HA. MR imaging features of intracranial primary CNS lymphoma in immune competent patients. Cancer Imaging 2014;14:22. 
32. Server A, Kulle B, Maehlen J, et al. Quantitative apparent diffusion coefficients in the characterization of brain tumors and associated peritumoral edema. Acta Radiol 2009;50:682-689.

33. Tong Z, Yamaki T, Harada K, Houkin K. In vivo quantification of the metabolites in normal brain and brain tumors by proton MR spectroscopy using water as an internal standard. Magnetic resonance imaging 2004;22:735-742.

34. Shimizu H, Kumabe T, Shirane R, Yoshimoto T. Correlation between choline level measured by proton MR spectroscopy and Ki-67 labeling index in gliomas. AJNR American journal of neuroradiology 2000;21:659-665.

35. Porto L, Kieslich M, Franz K, et al. MR spectroscopy differentiation between high and low grade astrocytomas: a comparison between paediatric and adult tumours. European journal of paediatric neurology : EJPN : official journal of the European Paediatric Neurology Society 2011;15:214-221.

36. Zaiss M, Kunz P, Goerke S, Radbruch A, Bachert P. MR imaging of protein folding in vitro employing nuclear-Overhauser-mediated 
saturation transfer. NMR in biomedicine 2013;26:1815-1822.

37. Yan K, Fu Z, Yang C, et al. Assessing Amide Proton Transfer (APT) MRI Contrast Origins in $9 \mathrm{~L}$ Gliosarcoma in the Rat Brain Using Proteomic Analysis. Molecular imaging and biology : MIB : the official publication of the Academy of Molecular Imaging 2015;17:479-487.

38. Scheidegger R, Wong ET, Alsop DC. Contributors to contrast between glioma and brain tissue in chemical exchange saturation transfer sensitive imaging at 3 Tesla. NeuroImage 2014;99:256-268.

39. Paech D, Burth S, Windschuh J, et al. Nuclear Overhauser Enhancement imaging of glioblastoma at 7 Tesla: region specific correlation with apparent diffusion coefficient and histology. PloS one 2015;10:e0121220.

40. Zaiss M, Windschuh J, Goerke S, et al. Downfield-NOE-suppressed amide-CEST-MRI at 7 Tesla provides a unique contrast in human glioblastoma. Magnetic resonance in medicine 2016.

41. Kurki T, Lundbom N, Kalimo H, Valtonen S. MR classification of brain gliomas: value of magnetization transfer and conventional imaging. Magnetic resonance imaging 1995;13:501-511. 
42. Sun PZ, Xiao G, Zhou IY, Guo Y, Wu R. A method for accurate pH mapping with chemical exchange saturation transfer (CEST) MRI. Contrast media \& molecular imaging 2016;11:195-202.

43. Harris RJ, Cloughesy TF, Liau LM, et al. pH-weighted molecular imaging of gliomas using amine chemical exchange saturation transfer MRI. Neuro-oncology 2015;17:1514-1524.

44. Bulik M, Jancalek R, Vanicek J, Skoch A, Mechl M. Potential of MR spectroscopy for assessment of glioma grading. Clinical neurology and neurosurgery 2013;115:146-153.

45. Longo DL, Bartoli A, Consolino L, et al. In Vivo Imaging of Tumor Metabolism and Acidosis by Combining PET and MRI-CEST pH Imaging. Cancer research 2016.

46. Guo Y, Zhou IY, Chan ST, et al. pH-sensitive MRI demarcates graded tissue acidification during acute stroke - $\mathrm{pH}$ specificity enhancement with magnetization transfer and relaxation-normalized amide proton transfer (APT) MRI. NeuroImage 2016;141:242-249.

47. Koslow SA, Claassen D, Hirsch WL, Jungreis CA. Gliomatosis cerebri: a case report with autopsy correlation. Neuroradiology 
1992;34:331-333. 


\section{Figure Legends}

Fig. 1 An 11-year-old boy diagnosed as anaplastic astrocytoma (Patient \#14). The tumor at the corpus callosum shows tiny enhancement on CE T1-weighted image (a). VOI for MRS was placed on the area showing the solid portion of tumor on T2-weighted image (source image of APT imaging) (b). The VOI was automatically transferred to the APT image by in-house MATLAB script (c). On APT image, the tumor shows increased signal in the corpus callosum. (d) LCModel analysis shows elevation of Cho and decreased NAA. These findings are compatible with high-grade brain tumor.

Fig. 2 A 35-year-old male diagnosed as oligodendroglioma (Patients \#3). The tumor shows no enhancement on CE T1-weighted image (a). VOI for MRS was placed on the area showing the solid portion of tumor on T2-weighted image (source image of APT imaging) (b). On APT image, the tumor in the left frontal lobe shows similar signal intensity to that of the contralateral normal white matter (c). LCModel analysis shows moderate level of Cho and NAA (d). These findings are compatible with low-grade brain tumor. 
Fig. 3 Receiver-operating-characteristics curve analysis for grading brain tumor with APT imaging, MRS and conventional MR imaging. ROC curves illustrated the performance of contrast enhancement, Cho, Cho/Cr, $\mathrm{APT}_{\text {mean }}$ and $\mathrm{APT}_{90} \cdot \mathrm{APT}_{\text {mean }}$ cutoff of 2.72 offered the best combination of sensitivity (72.7\%) and specificity (90\%) in the characterization of high-grade gliomas, while the $\mathrm{APT}_{90}$ cut-off of 6.61 demonstrated the optimal combination of sensitivity (90.9\%) and specificity (70\%). Cho/Cr threshold value of 0.50 generated the best combination of sensitivity (100\%) and specificity (93.8\%) in the discrimination of high- and low-grade tumors. All the imaging parameters above except Cho demonstrated higher AUC values than that of contrast enhancement (AUC was 0.65). 

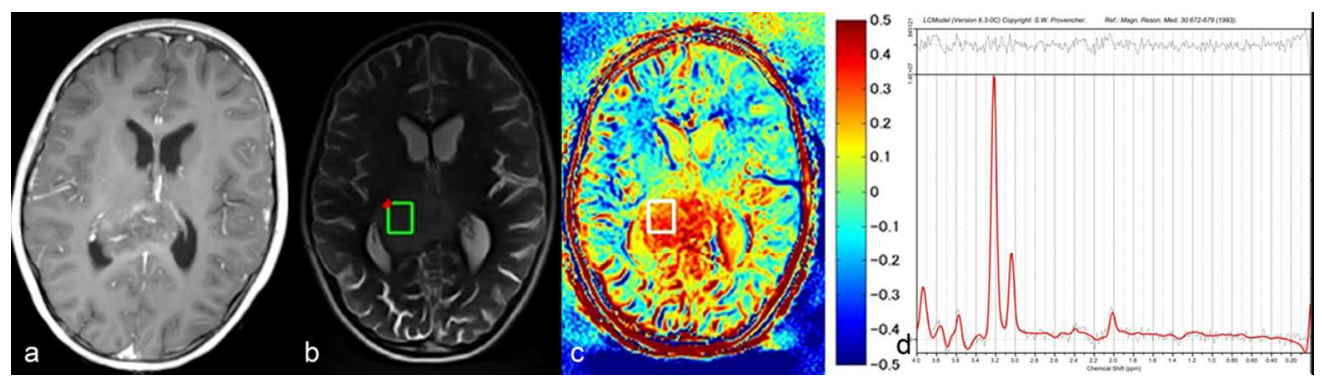

Fig. 1. An 11-year-old boy diagnosed as anaplastic astrocytoma (Patient \#14). The tumor at the corpus callosum shows tiny enhancement on CE T1-weighted image (a). VOI for MRS was placed on the area showing the solid portion of tumor on T2-weighted image (source image of APT imaging) (b). The VOI was automatically transferred to the APT image by in-house MATLAB script (c). On APT image, the tumor shows increased signal in the corpus callosum. (d) LCModel analysis shows elevation of Cho and decreased NAA. These findings are compatible with high-grade brain tumor.

$$
108 \times 30 \mathrm{~mm}(300 \times 300 \text { DPI })
$$




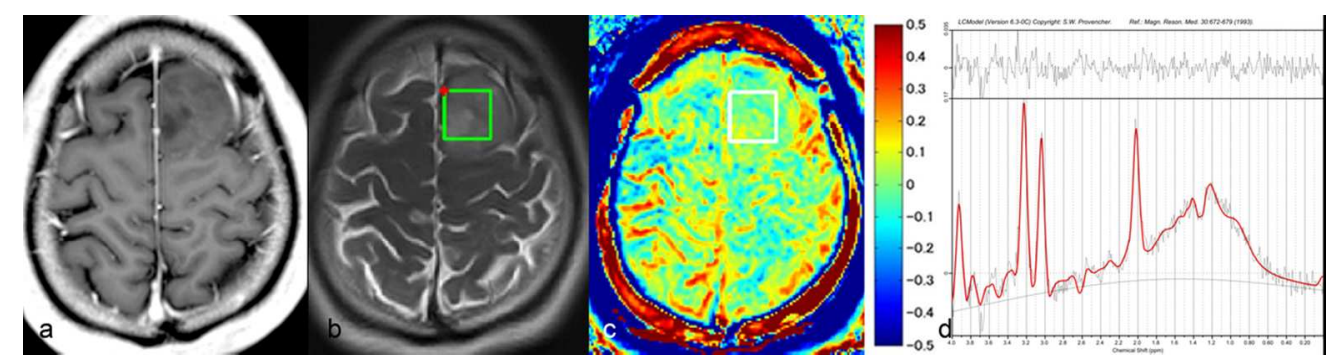

Fig. 2. A 35-year-old male diagnosed as oligodendroglioma (Patients \#3). The tumor shows no enhancement on CE T1-weighted image (a). VOI for MRS was placed on the area showing the solid portion of tumor on T2-weighted image (source image of APT imaging) (b). On APT image, the tumor in the left frontal lobe shows similar signal intensity to that of the contralateral normal white matter (c). LCModel analysis shows moderate level of Cho and NAA (d). These findings are compatible with low-grade brain tumor.

$108 \times 28 \mathrm{~mm}(300 \times 300 \mathrm{DPI})$ 
Fig. 3. Receiver-operating-characteristics curve analysis for grading brain tumor with APT imaging, MRS and conventional MR imaging. ROC curves illustrated the performance of contrast enhancement, Cho, Cho/Cr, $\mathrm{APT}_{\text {mean }}$ and $\mathrm{APT}_{90}$. $\mathrm{APT}_{\text {mean }}$ cutoff of 2.72 offered the best combination of sensitivity $(72.7 \%)$ and specificity $(90 \%)$ in the characterization of high-grade gliomas, while the APT 90 cut-off of 6.61 demonstrated the optimal combination of sensitivity $(90.9 \%)$ and specificity $(70 \%)$. Cho/Cr threshold value of 0.50 generated the best combination of sensitivity (100\%) and specificity $(93.8 \%)$ in the discrimination of high- and lowgrade tumors. All the imaging parameters above except Cho demonstrated higher AUC values than that of contrast enhancement (AUC was 0.65).

$108 \times 79 \mathrm{~mm}(300 \times 300$ DPI $)$ 